\title{
Acute postoperative pain management: focus on iontophoretic transdermal fentanyl
}

\author{
Consalvo Mattia \\ Flaminia Coluzzi \\ ICOT-Polo Pontino, Department of \\ Anesthesia, Intensive Care Medicine \\ and Pain Therapy, University "La \\ Sapienza" Roma, Italy
}

\begin{abstract}
Despite progress in the management of chronic pain, acute pain remains an issue for many postoperative patients. Although patient-controlled analgesia (PCA) has demonstrated efficacy and patient satisfaction, current techniques using intravenous (IV) administration present limitations, including the risk of programming errors and the potential to limit patient mobility due to pumps, lines, and tubing. The patient-controlled fentanyl hydrochloride ( $\mathrm{HCl})$ iontophoretic transdermal system (fentanyl ITS) was designed to address these concerns. Fentanyl ITS is an innovative, needle-free, self-contained drug-delivery system that uses iontophoretic technology to deliver fentanyl through the skin by application of a low-intensity electrical field. The results of several clinical studies are presented in this review. In three phase 3 placebo-controlled trials, fentanyl ITS was shown to be superior to placebo for the treatment of postoperative pain following major abdominal, orthopedic, and thoracic surgery. The results of one active-comparator phase 3 trial demonstrated comparable safety and efficacy with a standard morphine IV PCA dosing regimen, without significant difference in the side effect profile. Fentanyl ITS represents a safe, easy to use, non-invasive, and convenient alternative to current acute postoperative pain management modalities.
\end{abstract}

Keywords: patient-controlled analgesia, transdermal, fentanyl, iontophoresis, postoperative pain, needle-free

\section{Introduction}

Despite progress in pain management, moderate-to-severe acute postoperative pain remains a problem, and has been formally recognized as such by several associations and societies worldwide, including the Agency for Health Care Policy and Research (AHCPR; US), the International Association of the Study of Pain, and the Royal College of Surgeons (UK) (RCSCA 1990; AHCPR 1992; IASP 1992; Stephens et al 2003). According to the AHCPR, the ethical obligation to manage pain and relieve the patient's suffering is at the core of a healthcare professional's commitment, and anything harmful to the patient, including postoperative pain, should be minimized or prevented if possible (AHCPR 1992). Acute pain has been associated with thromboembolic and pulmonary complications, additional time spent in the hospital, hospital readmission for further pain management, impairment in quality of life, and the potential to develop chronic pain (ASA et al 2004). Acute pain not only adds undue physical and emotional stress to the patient, but it can also lead to increased healthcare costs (Stephens et al 2003)

Patient-controlled analgesia (PCA) refers to a method of pain control that allows patients to self-administer small doses of an analgesic agent as required. Following titration to comfort (a score of $\leq 4$ on a scale $0-10 ; 0=$ no pain, $10=$ worst possible pain) using a loading dose of an opioid administered intravenously, PCA allows the patient to control pain through self-administered, small, intermittent doses of an analgesic drug as needed (Grass 2005). A study of 200 postsurgical patients demonstrated a high level of patient satisfaction with PCA, noting that control over 
pain was its most clinically significant feature (top response by $50.5 \%$ of patients) (Chumbley et al 1998). Furthermore, there is some evidence of a decreased risk of postoperative pulmonary complications associated with PCA (Walder et al 2001). Intravenous (IV) PCA using opioids is one of the most common methods of providing postoperative analgesia for the treatment of acute pain (Grass 2005). Based on a meta-analysis of 32 randomized, controlled trials, intravenous opioid PCA provides better analgesia than conventional parenteral opioid regimens, without an increase in opioid-related side effects (Walder et al 2001). While patient-controlled epidural analgesia (PCEA) offers many of the same benefits of IV PCA, patient preference study results indicated that patients prefer IV PCA to PCEA (Sinatra RS et al 2002).

One of the most common PCA modalities for the management of acute postoperative pain is morphine sulfate IV PCA. Although this modality has dramatically improved postoperative pain management, there are limitations and potential safety concerns that may restrict its use (Viscusi and Schechter 2006). Nearly one quarter of the errors that commonly occur in post-anesthesia care units (PACUs) involve an improper dose of a medication, and PCA is one of the problem areas identified (Hicks et al 2004). The most significant risk when using PCA pumps is accidental overmedication leading to narcotic-induced respiratory depression. These pumps require programming by the clinician, introducing the possibility of complications, or even death, due to programming errors (Anonymous 1997; Brown et al 1997; Eade 1997; Vicente et al 2003). Additional safety issues include possible drug product confusion, device design flaws, inadequate staff training, prescription errors, and complications such as IV-related phlebitis (Campbell 1998; ISMP 2003). A recent study of 122 IV drug preparations found that $4(3 \%)$ had potentially severe errors in preparation and administration, $38(31 \%)$ had potentially moderate errors, and $16(13 \%)$ had potentially minor errors (Taxis and Barber 2004). PCA medical errors can be dangerous or even fatal (Cohen and Smetzer 2005), and the risk of errors associated with currently available modalities may limit their utility.

Furthermore, the IV PCA system requires a pump, line, tubing, power cables, and pole. These are not only time consuming to set up and maintain, but may also reduce patient mobility and interfere with physical therapy (Viscusi 2004). In a recent study ( $N=77)$, the unwieldiness of this set up was one the most frequent reasons for IV PCA discontinuation (15.6\%), second only to stopping treatment because the patient no longer experienced pain $(51.9 \%)$ (Chen et al 2001). Several new technologies offering alternative routes for PCA administration are being developed to address the limitations of current PCA techniques using IV administration.

The patient-controlled fentanyl $\mathrm{HCl}$ iontophoretic transdermal system (fentanyl ITS; IONSYS ${ }^{\mathrm{TM}}$, Janssen Pharmaceutica NV, Beerse, Belgium) (Figure 1) is a needlefree, self-contained drug-delivery system that uses iontophoretic technology to deliver fentanyl directly through the skin by application of a low-intensity electrical field (Chelly 2005). This review focuses on the technology, pharmacology, clinical efficacy, and safety of fentanyl ITS.

\section{FentanyI ITS: technology and design Technology}

The fentanyl ITS uses non-invasive iontophoretic technology - based on the electrotransport principle that similar charges repel each other - to deliver fentanyl through the skin by application of an external electrical field. A battery is used to generate a current that flows from the anode to the cathode, forming a current loop between the system and the patient. As the anode surface becomes increasingly positively charged, the similarly positively charged drug molecules of fentanyl are forced out of the system across the skin. In addition to fentanyl, this process has been used for the transdermal delivery of a number of ionizable drugs such as lidocaine (Bezzant et al 1988; Galinkin et al 2002; Rose et al 2002; Kearns et al 2003), morphine (Ashburn et al 1992; Stephen et al 1994), and corticosteroids (Bertolucci 1982; Nirschl et al 2003).

Although morphine sulfate is the most commonly used opioid for IV PCA, the properties of fentanyl make it an optimal candidate for iontophoretic drug delivery. Among the ideal properties of a drug for transdermal delivery are low molecular mass and high lipid solubility (Barry 2001). Compared with morphine sulfate, fentanyl $\mathrm{HCl}$ has a lower molecular weight (372 Da vs $758 \mathrm{Da}$ for morphine sulfate) and a higher lipophilicity (Peng and Sandler 1999). Fentanyl is approximately 100 times as potent as morphine (Bovill 1987), enters the central nervous system approximately 133 times as easily, and is metabolized to inactive metabolites (unlike morphine, which is metabolized to active metabolites that may be associated with neurotoxicity) (Peng and Sandler 1999; VHA 2004). Combined with its rapid induction of analgesia and intermediate duration of action 
(terminal elimination half-life of $2-5 \mathrm{~h}$ ) (Peng and Sandler 1999), these factors make fentanyl an ideal opioid for acute postoperative pain management via iontophoresis. Sufentanil could also be considered for administration via the transdermal route (Sebel et al 1987).

The optimal dosage of fentanyl for use with iontophoretic technology was determined based upon results of a randomized, double-blind, multicenter study conducted to evaluate the efficacy and safety of 3 doses of fentanyl $(20 \mu \mathrm{g}$, $40 \mu \mathrm{g}$, or $60 \mu \mathrm{g}$ delivered intravenously at a constant rate over 10 minutes with a maximum of 6 doses per hour) in 150 patients after major surgery (Camu et al 1998). The primary efficacy endpoint was assessed by the number of positive responders, defined as a patient's global assessment score of "very good" or "excellent," plus the absence of severe adverse effects. Secondary endpoints included pain

A)

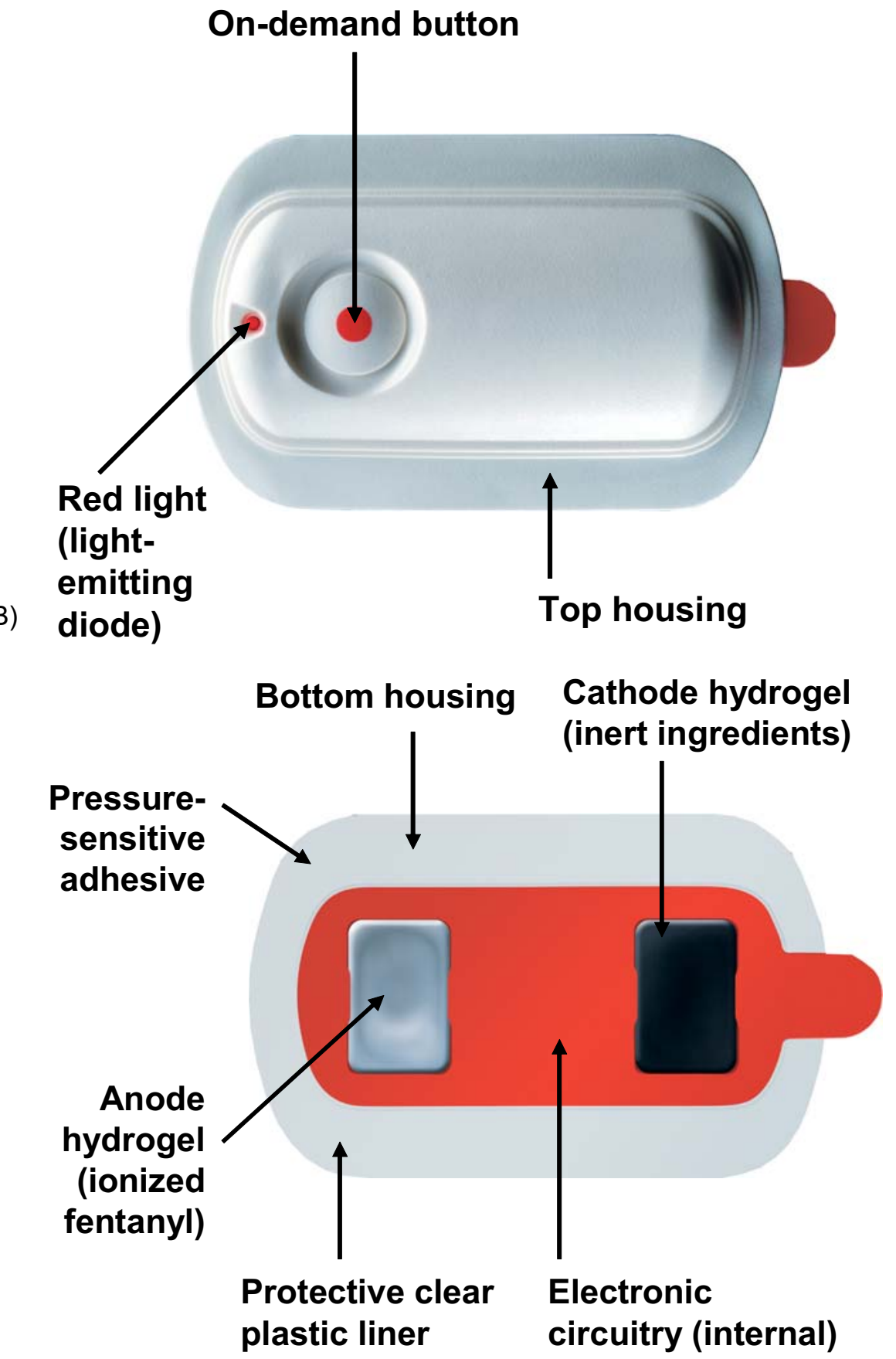

Figure I The fentanyl hydrochloride iontophoretic transdermal system (fentanyl ITS). 
intensity, the number of valid and invalid demand doses (invalid demand doses were defined as requests in excess of the 6 doses/hour limit), and the investigator ratings. Patients in the $60 \mu \mathrm{g}$ group had a significantly higher positive response rate (68\%) compared with those of the $20 \mu \mathrm{g}$ group (42\%; $\mathrm{p}=0.011)$. However, there was no significant difference between the $40 \mu \mathrm{g}$ group $(52 \%)$ and the $60 \mu \mathrm{g}$ or $20 \mu \mathrm{g}$ groups. The investigator ratings paralleled those of patients. The number of invalid demands (indicating that patients were not receiving adequate pain relief [Camu et al 1998]) were significantly higher for the $20 \mu \mathrm{g}$ group (124 vs 74 [40 $\mu$ g group] and 85 [60 $\mu$ g group]; $<<0.05)$. Patients in the $60 \mu \mathrm{g}$ group had significantly less pain at rest than those in the $20 \mu \mathrm{g}$ and $40 \mu \mathrm{g}$ groups $(\mathrm{p}=0.003$ and $\mathrm{p}=0.03$, respectively), and significantly less pain during movement than those in the $20 \mu \mathrm{g}$ group ( $\mathrm{p}=0.003$ ). Although the $40 \mu \mathrm{g}$ and $60 \mu \mathrm{g}$ fentanyl doses provided equal analgesia based on the number of invalid demands, the $60 \mu \mathrm{g}$ dose was associated with an increased risk of bradypnea. Taken together, these data suggest that the $40 \mu \mathrm{g}$ dose of fentanyl provided the best combination of efficacy and safety for fentanyl ITS.

The amount of drug delivered via an iontophoretic system is correlated with the magnitude of the total electrical current (Gupta, Bernstein, et al 1998; Gupta, Southam, et al 1998; Gupta et al 1999). To determine the optimal current for fentanyl ITS, a randomized, open-label study $(n=36)$ was conducted to investigate several fentanyl ITS prototypes with different current magnitude/anode area combinations, including $100 \mu \mathrm{A} / 1.38 \mathrm{~cm}^{2}, 140 \mu \mathrm{A} / 2.72 \mathrm{~cm}^{2}, 170 \mu \mathrm{A} /$ $2.75 \mathrm{~cm}^{2}, 200 \mu \mathrm{A} / 2.75 \mathrm{~cm}^{2}$, and $230 \mu \mathrm{A} / 2.75 \mathrm{~cm}^{2}$ (Sathyan, Jaskowiak, et al 2005). The treatments from each prototype were administered as 2 consecutive 10-minute doses during the first 20 minutes of each hour for $23.33 \mathrm{~h}$, with a single 10 -minute dose at $23 \mathrm{~h}$. The amount of fentanyl absorbed from fentanyl ITS was proportional to current for each 10minute, on-demand dose and was estimated to be $24.8( \pm 5.2) \mu \mathrm{g}, \quad 35.1( \pm 12.1) \mu \mathrm{g}, \quad 39.5( \pm 10.9) \mu \mathrm{g}$, $49.7( \pm 8.8) \mu \mathrm{g}$, and $53.9( \pm 12.8) \mu \mathrm{g}$ for $100 \mu \mathrm{A} / 1.38 \mathrm{~cm}^{2}$, $140 \mu \mathrm{A} / 2.72 \mathrm{~cm}^{2}, 170 \mu \mathrm{A} / 2.75 \mathrm{~cm}^{2}, 200 \mu \mathrm{A} / 2.75 \mathrm{~cm}^{2}$, and $230 \mu \mathrm{A} / 2.75 \mathrm{~cm}^{2}$, respectively. Based on these results, $170 \mu \mathrm{A}$ was considered the optimal current for delivery of the required $40 \mu \mathrm{g}$ of fentanyl.

\section{Design}

The fentanyl ITS consists of 2 components housed within a single, compact, self-contained system that may be applied to the patient's upper outer arm or chest. The electromechanical component is located within the top housing and contains a printed circuit board and integrated circuit, transducer, capacitors, and lithium battery, as well as a recessed on-demand button and red light-emitting diode (LED). The drug component of the system consists of an anode hydrogel containing fentanyl $\mathrm{HCl}$ and a cathode hydrogel that contains no pharmacologically active ingredients. An adhesive on the outer edges of the unit allows the system to be attached to a patient's skin without the requirement for venous access. This completely selfcontained, easy-to-use system eliminates the need for the pump, line, tubing, power cables, and pole associated with the use of IV PCA.

Based on dose-finding and electrical current studies (Camu et al 1998; Gupta, Bernstein, et al 1998; Gupta, Southam, et al 1998; Gupta et al 1999; Sathyan, Jaskowiak, et al 2005), the fentanyl ITS has been pre-programmed to deliver fentanyl $\mathrm{HCl} 40 \mu \mathrm{g}$ over a 10 min period with each system, providing a maximum of 6 doses per hour. When the patient presses the recessed on-demand button twice within 3 seconds, an electrical current passes through the fentanyl-containing anode hydrogel. The fentanyl is then ionized, and the positively charged drug molecules are repelled from the similarly positively charged anode surface. A system-initiated lockout prevents the patient from activating the system for additional drug delivery during the 10 -minute infusion. The system operates for $24 \mathrm{~h}$ or delivers up to 80 doses, whichever occurs first. After this point, the system is inactivated and unresponsive to additional requests for medications. Therefore, the system has to be removed at the conclusion of the $24 \mathrm{~h}$ dosing period and a second system applied if continued analgesia is required.

A small light-emitting diode (LED) and audible beeps communicate the system's dosing status. When the patient initiates drug delivery by pressing the recessed on-demand button, an audible beep and a red light from the LED indicate that a dose has been initiated. After each dose is delivered, the LED flashes to indicate the cumulative number of doses a patient has received, with each flash representing up to 5 doses. Healthcare providers may query the system to display the approximate number of doses delivered at any time. Each LED flash represents 1-5 total doses administered, so 2 flashes represent 6-10 doses, 3 flashes represent 11-15 doses, and 16 flashes represent 76-80 doses administered. 


\section{Pharmacokinetics of fentanyl ITS Dosing frequency}

Three randomized, open-label studies were performed to determine the effect of dosing frequency on the pharmacokinetics of fentanyl ITS (Sathyan, Zomordi, et al 2005). In the first study, 30 subjects received 3 fentanyl ITS $25 \mu \mathrm{g}$ treatments, including 2 sequential doses hourly for $23.33 \mathrm{~h}(1 \mathrm{~A}), 6$ sequential doses every $3 \mathrm{~h}$ for $22 \mathrm{~h}(1 \mathrm{~B})$, and 72 doses continuously for $12 \mathrm{~h}(1 \mathrm{C})$. All doses were delivered on demand over 10 minutes, and the area under the serum concentration-versus-time curves for a single dose $\left(\mathrm{AUC}_{\mathrm{n}}\right.$ ) were calculated by dividing $\mathrm{AUC}_{23-24}$ (for $1 \mathrm{~A}$ ), $\mathrm{AUC}_{21-24}(1 \mathrm{~B})$, and $\mathrm{AUC}$, (1C) by the number of doses in each dosing interval $(2,6$, or 72 for $1 \mathrm{~A}, 1 \mathrm{~B}$, and $1 \mathrm{C}$, respectively). The $\mathrm{AUC}_{\mathrm{n}}$ for $1 \mathrm{~A}$ and $1 \mathrm{~B}$ were similar $(0.374 \mu \mathrm{g} \cdot \mathrm{h} / \mathrm{L}$ and $0.365 \mu \mathrm{g} \cdot \mathrm{h} / \mathrm{L}$, respectively), suggesting that the bioavailability of fentanyl is independent of dosing frequency. Furthermore, the $\mathrm{AUC}_{\mathrm{n}}$ for $1 \mathrm{C}$, which had a shorter treatment duration than $1 \mathrm{~A}$ and $1 \mathrm{~B}$, was significantly lower than $1 \mathrm{~A}$ or $1 \mathrm{~B}(0.314 \mu \mathrm{g} \cdot \mathrm{h} / \mathrm{L} ; \mathrm{p}=0.001)$, suggesting that bioavailability increases over time. The second study paralleled the first study, but investigated a $40 \mu \mathrm{g}$ dose rather than a $25 \mu \mathrm{g}$ dose (Sathyan, Zomordi, et al 2005). The conclusions of the second study were the same - the bioavailability of fentanyl delivered via fentanyl ITS increased as a function of time and was independent of dosing frequency (Sathyan, Zomordi, et al 2005).

In the third study, 20 subjects received 4 fentanyl ITS $40 \mu \mathrm{g}$ treatments, including 6 doses over $1 \mathrm{~h}(3 \mathrm{~A}), 18$ doses over $3 \mathrm{~h}$ (3B), 36 doses over $6 \mathrm{~h}(3 \mathrm{C})$, and 80 doses over $13.33 \mathrm{~h}$ (3D) (Sathyan, Zomordi, et al 2005). Based on the dose-normalized AUC data, $41 \%$ of each nominal $40 \mu \mathrm{g}$ dose was absorbed into the systemic circulation in the first hour of treatment, and increased to nearly $100 \%$ after $10 \mathrm{~h}$. This delay in absorption over time is not clinically relevant (since patients self-administer medication according to their pain level) and may be due to alterations in the electrical conduction properties of the skin that may occur as it adjusts to the current input from fentanyl ITS (Sathyan, Zomordi, et al 2005).

\section{Multi-day administration and comparison to fentanyl IV PCA}

Another study investigated the effects of single- and multiple-day administration on the pharmacokinetics of fentanyl delivered via fentanyl ITS, and compared the pharmacokinetics of fentanyl ITS versus fentanyl IV PCA
(Sathyan, Jaskowiak, et al 2005). In the administration study, 28 subjects first received a single-day administration of two 10-minute doses of fentanyl $40 \mu \mathrm{g}$ from the fentanyl ITS every $4 \mathrm{~h}$ for $20 \mathrm{~h}$. This treatment was followed by a multipleday administration of two 10-minute doses every $4 \mathrm{~h}$ for $68 \mathrm{~h}$, with a new fentanyl ITS placed on a new skin site at $0 \mathrm{~h}, 24 \mathrm{~h}$, and $48 \mathrm{~h}$. Based on corrected AUC values of $0.40 \mu \mathrm{g} \cdot \mathrm{h} / \mathrm{L}$ and $0.54 \mu \mathrm{g} \cdot \mathrm{h} / \mathrm{L}$ for the single- and multipleday treatments, respectively, there was no significant difference between study groups $(\mathrm{p}=0.133)$, and multipleday administration did not alter the pharmacokinetics of fentanyl ITS (Sathyan, Jaskowiak, et al 2005). The results of the study comparing the pharmacokinetics of fentanyl ITS with fentanyl IV PCA ( $80 \mu \mathrm{g}$ of IV fentanyl over 20 minutes compared with 2 consecutive 10-minute doses of $40 \mu \mathrm{g}$ each from the fentanyl ITS) demonstrated that both modalities display similar pharmacokinetic profiles (Table 1). Additionally, the between-subject variability and decline in serum fentanyl concentration after completion of treatment were similar for both fentanyl ITS and fentanyl IV PCA modalities, suggesting that the use of fentanyl ITS is not associated with any greater variability in serum fentanyl concentration than would be seen with fentanyl IV PCA. After the fentanyl ITS is removed, the decline in serum fentanyl concentration is similar to IV PCA, suggesting negligible drug depot in the skin (Sathyan, Jaskowiak, et al 2005).

\section{Effects of application site and subject demographics}

The effects of application site and subject demographics on pharmacokinetics were also examined (Gupta et al 2005). The effect of application site was examined in a randomized

Table I Pharmacokinetics of fentanyl hydrochloride iontophoretic transdermal system (ITS) versus fentanyl intravenous patient-controlled analgesia (IV PCA) (Sathyan, Jaskowiak, et al 2005) ${ }^{a}$

\begin{tabular}{lll}
\hline & $\begin{array}{l}\text { Fentanyl } \\
\text { ITS 40 } \boldsymbol{\mu g} \\
(\mathbf{n = 3} \mathbf{~ I})\end{array}$ & $\begin{array}{l}\text { Fentanyl } \\
\text { IV PCA 80 } \mathbf{\mu g} \\
(\mathbf{n = 3} \text { I) }\end{array}$ \\
\hline $\mathrm{C}_{\max }(\mu \mathrm{g} / \mathrm{L})$ & 1.37 & 1.82 \\
$\mathrm{t}_{\max }(\mathrm{h})$ & 0.65 & 0.58 \\
$\mathrm{t}_{1 / 2}(\mathrm{~h})$ & 11.0 & 12.6 \\
$\mathrm{AUC}_{23-24}(\mu \mathrm{g} \cdot \mathrm{h} / \mathrm{L})$ & 1.23 & 1.34 \\
\hline
\end{tabular}

a $80 \mu$ g of fentanyl was delivered via IV PCA over 20 min compared with 2 consecutive $10 \mathrm{~min}$ doses of $40 \mu \mathrm{g}$ fentanyl each from the fentanyl ITS.

Abbreviations: AUC, area under serum concentration curve; $C_{\max }$, peak serum concentration; $t_{\max }$, time to peak serum concentration; $t_{1 / 2}$, half-life. 
study in which 34 subjects placed fentanyl ITS on the chest, upper outer arm, or lower inner arm. The results demonstrated a significantly lower $\mathrm{AUC}_{23-24}$ (AUC for 23$24 \mathrm{~h})$ with application to the lower inner $\operatorname{arm}(0.757 \mu \mathrm{g} \cdot \mathrm{h} /$ L) compared with the upper outer $\operatorname{arm}(1.033 \mu \mathrm{g} \cdot \mathrm{h} / \mathrm{L}$; $\mathrm{p}=0.005)$ or chest $(1.015 \mu \mathrm{g} \cdot \mathrm{h} / \mathrm{L} ; \mathrm{p}=0.013)$. To investigate the effects of subject demographics on the pharmacokinetics of fentanyl ITS, 70 subjects received fentanyl ITS $40 \mu \mathrm{g} 3$ times during the first 30 minutes of each hour for $3 \mathrm{~h}$ and were evaluated according to age, body weight, gender, and ethnicity (Gupta et al 2005). The results demonstrated that none of these demographic characteristics affected the pharmacokinetics of fentanyl delivered via fentanyl ITS.

\section{Efficacy of fentanyl ITS}

The efficacy of fentanyl ITS in treating acute postoperative pain was first established in three phase 3 double-blind placebo-controlled clinical trials (Brown et al 1998; Chelly et al 2004; Viscusi et al 2006). The first of these trials was a $3: 1$ randomization study in which patients received either $40 \mu \mathrm{g}$ of fentanyl $\mathrm{HCl}$ via fentanyl ITS over 10 minutes $(n=76)$ or placebo $(n=25)$ for the management of moderateto-severe acute postoperative pain (Brown et al 1998). Patients who received fentanyl ITS had lower dropout rates (7\% vs 38\%; $\mathrm{p}=0.001$ ) (Figure 2 ) and lower pain intensity scores on a $100 \mathrm{~mm}$ visual analog scale $(0 \mathrm{~mm}=$ no pain, $100 \mathrm{~mm}=$ worst possible pain; 28 vs $37 ; \mathrm{p}=0.016)$ than control patients. The fentanyl ITS was also ranked as an "excellent" method of pain management by significantly more patients $(65 \%$ vs $19 \%$; $<<0.001)$ and investigators (62\% vs $19 \% ; \mathrm{p}<0.001)$ compared with placebo.

A second, larger 3:1 randomization study assessed the efficacy of fentanyl ITS in 189 patients who underwent major abdominal, orthopedic, or thoracic surgery (Chelly et al 2004). Patients received a maximum of six $40 \mu \mathrm{g} 10-$ minute doses of fentanyl ITS per hour for up to $24 \mathrm{~h}$ or 80 doses, whichever occurred first. Fewer patients who received fentanyl ITS withdrew from the trial because of inadequate analgesia after at least $3 \mathrm{~h}$ of treatment compared with placebo (25.4 \% vs 40.4\%; p<0.05) (Figure 2). Patients treated with fentanyl ITS also reported lower (better) last pain intensity scores on a $100 \mathrm{~mm}$ visual analog scale $(0 \mathrm{~mm}$ $=$ no pain, $100 \mathrm{~mm}=$ worst possible pain; 30.9 vs 40.8 ; $\mathrm{p}=0.047$ ). The global assessment of method of pain control was better for fentanyl ITS than placebo based on a 4-point categorical scale in which $1=$ "poor" and $4=$ "excellent" as judged by patients ( 3.0 vs $2.6 ; \mathrm{p}=0.047)$ and investigators (3.1 vs 2.6; $p=0.007$ ). One limitation of this study, which could have minimized the observed efficacy, was that a greater proportion of patients $(5: 1)$ who received fentanyl ITS had high ( $\geq 75$ on a $100 \mathrm{~mm}$ visual analog scale) baseline pain intensity scores, even though mean baseline pain scores between treatment groups were similar. This could have resulted in higher relative patient withdrawal because of

\section{$\square$ Fentanyl ITS $\square$ Placebo}

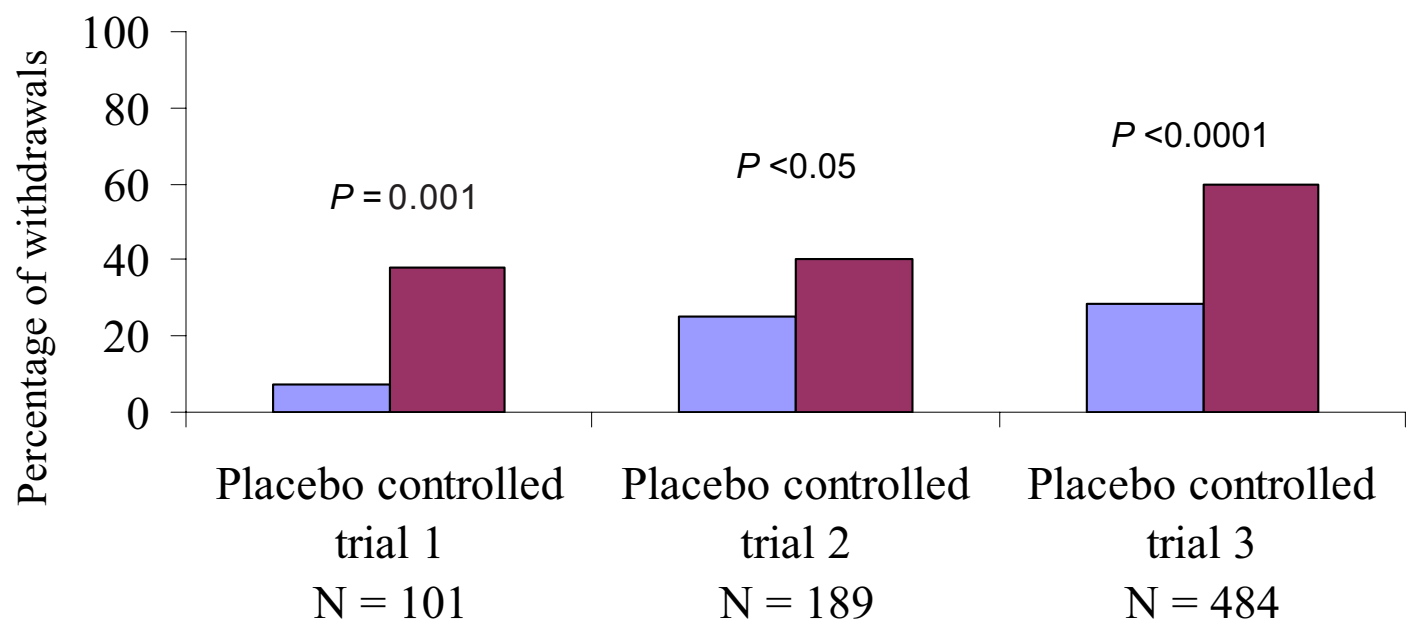

\section{Trial}

Figure 2 Withdrawals due to inadequate analgesia in placebo-controlled trials (Brown et al 1998; Chelly et al 2004; Viscusi et al 2006).

Abbreviations: fentanyl ITS, fentanyl hydrochloride iontophoretic transdermal system. 


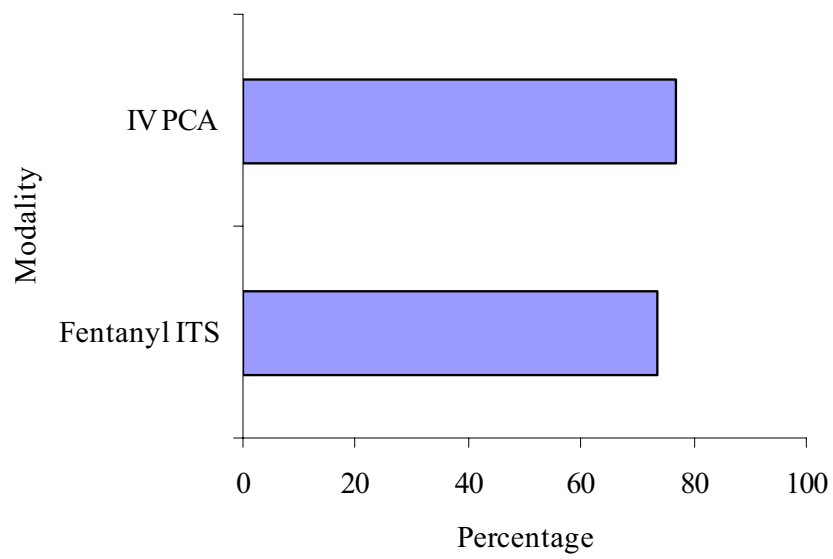

Figure 3 Percentage of patients rating pain control as "good" or "excellent" in the active-controlled trial (Viscusi et al 2004).

Abbreviations: fentanyl ITS, fentanyl hydrochloride iontophoretic transdermal system; IV, intravenous; PCA, patient-controlled analgesia.

inadequate analgesia in patients receiving fentanyl ITS compared with placebo. A third phase 3 study evaluating the efficacy of fentanyl $\mathrm{HCl}$ versus placebo addressed this limitation by setting a maximum baseline pain score for enrolled patients (Viscusi et al 2006).

The findings of the largest placebo-controlled trial for fentanyl ITS were published in 2006 (Viscusi et al 2006). In this study, 484 patients were treated with fentanyl ITS $(n=244)$ or placebo $(n=240)$ after major surgery. To minimize disproportionate baseline pain scores between groups, only patients with pain scores $<5$ on an 11-point scale $(0=$ no pain, $10=$ worst possible pain $)$ were enrolled following initial IV opioid administration. Half as many patients treated with fentanyl ITS withdrew from the study because of inadequate analgesia compared with placebo $(28.7 \%$ vs $60 \% ; p<0.0001)$ (Figure 2$)$, and nearly twice as many patients were "very satisfied" with their pain management compared with placebo $(61.5 \%$ vs $32.1 \%)$. The mean pain intensity scores of fentanyl-treated patients were significantly lower (better) throughout the $24 \mathrm{~h}$ treatment schedule, including the last pain intensity score on an 11point scale ( 3.5 vs $5.4 ; \mathrm{p}<0.0001)$. The overall method of pain control was also described as "excellent" in those who received fentanyl ITS by significantly more patients $(39.8 \%$ vs $14.6 \%$; $<0.0001)$ and investigators $(47.5 \%$ vs $20.8 \%$; $\mathrm{p}<0.0001$ ) compared with placebo.

In 2004, the results of the first study comparing the efficacy of fentanyl ITS with morphine IV PCA were published (Viscusi et al 2004). In this prospective, parallelgroup trial, 636 patients were assigned randomly to receive IV morphine (1 $\mathrm{mg}$ dose as a bolus, with a $5 \mathrm{~min}$ lockout and a maximum of $10 \mathrm{mg} / \mathrm{h})$ by PCA pump $(\mathrm{n}=320)$ or fentanyl ITS ( $40 \mu \mathrm{g}$ treatment over $10 \mathrm{~min}$; $\mathrm{n}=316$ ) (Viscusi et al 2004). Most patients were female (72.5\%), white (73.7\%), and had undergone major abdominal, orthopedic, or thoracic surgery. Both modalities were therapeutically equivalent according to pain intensity scores at all assayed time points during the $24 \mathrm{~h}$ treatment period, and the distribution of patients' ratings of pain control as poor, fair, good, or excellent between treatment groups were not statistically different. Assessment of successful global pain management was determined by combining patients' ratings of pain control as good and excellent for each modality. These values were similar for fentanyl ITS and morphine IV PCA ( $73.7 \%$ and $76.9 \%$, respectively; $\mathrm{p}=0.36$ ) (Figure 3 ), further suggesting therapeutic equivalence between the 2 PCA systems.

\section{Safety and tolerability of fentanyl ITS}

To date, the safety and tolerability of fentanyl ITS have been examined in a total of 4 clinical studies (Brown et al 1998; Chelly et al 2004; Viscusi et al 2004, 2006). Adverse events associated with fentanyl ITS are similar to those reported with IV opioid administration, including nausea, vomiting, pruritus, headache, and mild-to-moderate dizziness. Nausea was the most common adverse event, with the incidence ranging between $26.6 \%$ and $67.5 \%$. In the active-comparator phase 3 trial, the incidence of nausea was similar between fentanyl ITS and IV morphine (Viscusi et al. 2004). Serious adverse events possibly related to fentanyl ITS included 1 case of severe urinary retention accompanied by moderate nausea and vomiting (Chelly et al 2004), 2 cases of ileus diagnosed after removal of fentanyl ITS (Viscusi et al 2006), and 1 report of severe confusion (Viscusi et al 2004). No patient treated with fentanyl ITS developed clinical respiratory depression (defined as the simultaneous occurrence of bradypnea [respiratory rate $<8$ breaths per minute] and excessive sedation) in any of the 4 trials $(n=778)$ (Brown et al 1998; Chelly et al 2004; Viscusi et al 2004, 2006). Application-site skin reactions, such as erythema, rash, itching, and vesicles, were also observed in patients treated with fentanyl ITS, but their incidence was lower than 7\% (Brown et al 1998; Chelly et al 2004; Gupta et al 2005; Viscusi et al 2006). Moreover, most of the application-site reactions were mild, resembling sunburn or tanning marks, and none required treatment (Viscusi et al 2006). 


\section{Conclusions}

Findings from several controlled clinical studies indicate that fentanyl ITS is safe and effective for the management of acute postoperative pain. The fentanyl ITS allows patients to maintain an acceptable level of pain control following titration to comfort with a loading dose of opioid (Viscusi et al 2004), while avoiding the first-pass effect and analgesic gaps associated with other pain management modalities. Patient satisfaction was demonstrated through the low number of withdrawals due to inadequate analgesia and the high percentage of patients rating pain control with fentanyl ITS as good or excellent. Furthermore, fentanyl ITS is the first needle-free, self-contained, patient-activated system developed for the management of acute postoperative pain, making it a safe, easy to use, and convenient modality of pain relief for both patients and healthcare professionals.

\section{Acknowledgment}

The authors gratefully acknowledge the editorial assistance of Michael Malia at MedErgy HealthGroup.

\section{References}

[AHCPR] Agency for Health Care Policy and Research. 1992. Acute pain management: operative or medical procedures and trauma, part 1. Agency for Health Care Policy and Research. Clin Pharm, 11:30931.

Anonymous. 1997. Abbott PCA Plus II patient-controlled analgesic pumps prone to misprogramming resulting in narcotic overinfusions. Health Devices, 26:389-91.

[ASA] American Society of Anesthesiologists Task Force on Acute Pain Management, Ashburn MA, Caplan RA, et al. 2004. Practice guidelines for acute pain management in the perioperative setting: an updated report by the American Society of Anesthesiologists Task Force on Acute Pain Management. Anesthesiology, 100:1573-81.

Ashburn MA, Stephen RL, Ackerman E, et al. 1992. Iontophoretic delivery of morphine for postoperative analgesia. J Pain Symptom Manage, 7:27-33.

Barry BW. 2001. Novel mechanisms and devices to enable successful transdermal drug delivery. Eur J Pharm Sci, 14:101-14.

Bertolucci LE. 1982. Introduction of antiinflammatory drugs by iontophoresis: double-blind study. J Orthop Sports Phys Ther, 4:1038.

Bezzant JL, Stephen RL, Petelenz TJ, et al. 1988. Painless cauterization of spider veins with the use of iontophoretic local anesthesia. $J \mathrm{Am}$ Acad Dermatol, 19:869-75.

Bovill JG. 1987. Which potent opioid? Important criteria for selection. Drugs, 33:520-30.

Brown CR, Moodie JE, Bisley EJ. Safety and efficacy of Transfenta in the treatment of post-operative pain: a double-blind, single-center, placebo-controlled trial. Presented at the 17th Annual Scientific Meeting of the American Pain Society; November 5-8, 1998, San Diego, Calif.

Brown SL, Bogner MS, Parmentier CM, et al. 1997. Human error and patient-controlled analgesia pumps. J Intraven Nurs, 20:311-16.

Campbell L. 1998. IV-related phlebitis, complications and length of hospital stay: 2. Br J Nurs, 7:1364-70, 1372.
Camu F, Van Aken H, Bovill JG. 1998. Postoperative analgesic effects of three demand-dose sizes of fentanyl administered by patient-controlled analgesia. Anesth Analg, 87:890-5.

Chelly JE, Grass J, Houseman TW, et al. 2004. The safety and efficacy of a fentanyl patient-controlled transdermal system for acute postoperative analgesia: a multicenter, placebo-controlled trial. Anesth Analg, 98:427-33.

Chelly JE. 2005. An iontophoretic, fentanyl $\mathrm{HCl}$ patient-controlled transdermal system for acute postoperative pain management. Expert Opin Pharmacother, 6:1205-14.

Chen PP, Chui PT, Ma M, et al. 2001. A prospective survey of patients after cessation of patient-controlled analgesia. Anesth Analg, 92:224-7.

Chumbley GM, Hall GM, Salmon P. 1998. Patient-controlled analgesia: an assessment by 200 patients. Anaesthesia, 53:216-21.

Cohen MR, Smetzer J. 2005. Patient-controlled analgesia safety issues. $J$ Pain Palliat Care Pharmacother, 19:45-50.

Eade DM. 1997. Patient-controlled analgesia - eliminating errors. Nurs Manage, 28:38-40.

Galinkin JL, Rose JB, Harris K, et al. 2002. Lidocaine iontophoresis versus eutectic mixture of local anesthetics (EMLA) for IV placement in children. Anesth Analg, 94:1484-8.

Grass JA. 2005. Patient-controlled analgesia. Anesth Analg, 101:S44-S61.

Gupta SK, Bernstein KJ, Noorduin H, et al. 1998. Fentanyl delivery from an electrotransport system: delivery is a function of total current, not duration of current. J Clin Pharmacol, 38:951-8.

Gupta SK, Hwang S, Southam M, et al. 2005. Effects of application site and subject demographics on the pharmacokinetics of fentanyl $\mathrm{HCl}$ patient-controlled transdermal system (PCTS). Clin Pharmacokinet, 44(suppl 1):25-32.

Gupta SK, Sathyan G, Phipps B, et al. 1999. Reproducible fentanyl doses delivered intermittently at different time intervals from an electrotransport system. J Pharm Sci, 88:835-41.

Gupta SK, Southam M, Sathyan G, et al. 1998. Effect of current density on pharmacokinetics following continuous or intermittent input from a fentanyl electrotransport system. J Pharm Sci, 87:976-81.

Hicks R, Becker S, Krenzischeck D, et al. 2004. Medication errors in the PACU: a secondary analysis of MEDMARX findings. $J$ PeriAnesth Nurs, 19:18-28.

[IASP] International Association of the Study of Pain, Task Force on Acute Pain. 1992. In: Ready LB EW, (ed). Management of acute pain: A practical guide. Seattle, WA: IASP Pr.

[ISMP] Institute of Safe Medication Practice. 2003. Institute of Safe Medication Practice (ISMP). Medication Safety Alert newsletter. Safety issues with patient-controlled analgesia: Part I - How errors occur [online]. Accessed 13 January 2005. URL: http://www.ismp.org/ MSAarticles/issue2.htm.

Kearns GL, Heacook J, Daly SJ, et al. 2003. Percutaneous lidocaine administration via a new iontophoresis system in children: tolerability and absence of systemic bioavailability. Pediatrics, 112:578-82.

Nirschl RP, Rodin DM, Ochiai DH, et al. 2003. Iontophoretic administration of dexamethasone sodium phosphate for acute epicondylitis. A randomized, double-blinded, placebo-controlled study. Am J Sports Med, 31:189-95.

Peng PW, Sandler AN. 1999. A review of the use of fentanyl analgesia in the management of acute pain in adults. Anesthesiology, 90:576-99.

[RCSCA] Royal College of Surgeons and College of Anaesthetists. 1990. Royal College of Surgeons of England and College of Anaesthetists Commission on the Provision of Surgical Services. Report of the Working Party on Pain After Surgery London: Royal College of Surgeons and College of Anaesthetists.

Rose JB, Galinkin JL, Jantzen EC, et al. 2002. A study of lidocaine iontophoresis for pediatric venipuncture. Anesth Analg, 94:867-71.

Sathyan G, Jaskowiak J, Evashenk M, et al. 2005. Characterisation of the pharmacokinetics of fentanyl $\mathrm{HCl}$ patient-controlled transdermal system (PCTS): effect of current magnitude and multiple-day dosing, and comparison with IV fentanyl administration. Clin Pharmacokinet, 44(suppl 1):7-15. 
Sathyan G, Zomordi K, Gidwani S, et al. 2005. The effect of dosing frequency on the pharmacokinetics of fentanyl $\mathrm{HCl}$ patient-controlled transdermal system (PCTS). Clin Pharmacokinet, 44(suppl 1):17-24.

Sebel PS, Barrett CW, Kirk CJ, et al. 1987. Transdermal absorption of fentanyl and sufentanil in man. Eur J Clin Pharmacol, 32:529-31.

Sinatra RS, Torres J, Bustos AM. 2002. Pain management after major orthopaedic surgery: current strategies and new concepts. $J$ Am Acad Orthop Surg, 10:117-29.

Stephen R, Miotti D, Bettaglio R, et al. 1994. Electromotive administration of a new morphine formulation: morphine citrate. Artif Organs, 18:461-5.

Stephens J, Laskin B, Pashos C, et al. 2003. The burden of acute postoperative pain and the potential role of the COX-2-specific inhibitors. Rheumatology (Oxford), 42(suppl 3):iii40-iii52.

Taxis K, Barber N. 2004. Incidence and severity of intravenous drug errors in a German hospital. Eur J Clin Pharmacol, 59:815-7.

[VHA] Veterans Health Administration. 2004. Veterans Health Administration, Department of Defense. Clinical practice guideline for the management of postoperative pain [online]. Accessed 28 December 2004. URL: http://www.oqp.med.va.gov/cpg/PAIN/ PAIN_GOL.htm.
Vicente KJ, Kada-Bekhaled K, Hillel G, et al. 2003. Programming errors contribute to death from patient-controlled analgesia: case report and estimate of probability. Can J Anaesth, 50:328-32.

Viscusi ER, Reynolds L, Chung F, et al. 2004. Patient-controlled transdermal fentanyl hydrochloride vs intravenous morphine pump for postoperative pain: a randomized controlled trial. JAMA, 291:133341.

Viscusi ER, Reynolds L, Tait S, et al. 2006. An iontophoretic fentanyl patient-controlled analgesic delivery system for postoperative pain: a double-blind, placebo-controlled trial. Anesth Analg, 102:188-94.

Viscusi ER, Schechter LN. 2006. Patient-controlled analgesia: finding a balance between cost and comfort. Am J Health Syst Pharm, 63:S3S13.

Viscusi ER. 2004. Emerging techniques in the treatment of postoperative pain. Am J Health Syst Pharm, 61(suppl 1):S11-S14.

Walder B, Schafer M, Henzi I, et al. 2001. Efficacy and safety of patientcontrolled opioid analgesia for acute postoperative pain. A quantitative systematic review. Acta Anaesthesiol Scand, 45:795-804. 
